\title{
Three-dimensional stability of the solar tachocline
}

\author{
R. Arlt $^{1}$, A. Sule ${ }^{1}$, and G. Rüdiger ${ }^{1,2}$ \\ 1 Astrophysikalisches Institut Potsdam, An der Sternwarte 16, Germany \\ e-mail: rarlt@aip.de \\ 2 Isaac Newton Institute for Mathematical Sciences, 20 Clarkson Road, Cambridge CB3 0EH, UK
}

Received 24 February 2005 / Accepted 20 May 2005

\begin{abstract}
The three-dimensional, hydrodynamic stability of the solar tachocline was investigated based on a rotation profile as a function of both latitude and radius. By varying the amplitude of the latitudinal differential rotation, we found linear stability limits at various Reynolds numbers by numerical computations. We repeated the computations with different latitudinal and radial dependences of the angular velocity. The stability limits are all higher than those previously found from two-dimensional approximations and higher than the shear expected in the Sun. It is concluded that any part of the tachocline which is radiative is hydrodynamically stable against small perturbations.
\end{abstract}

Key words. Sun: interior - instabilities - hydrodynamics

\section{Motivation}

Helioseismology has provided us with information on the internal rotation of the Sun. The angular velocity depends on latitude, as well as radius. The dependence is mainly a latitudinal one in the bulk of the convection zone, whereas the solar radiative core rotates nearly uniform with an angular velocity of the convection zone at about $30^{\circ}$ latitude. The transition from the differential rotation in the convection zone to the uniform rotation in the core is thin - probably thinner than $5 \%$ of the solar radius - and is called the tachocline.

The convection zone is thermally overcritical and the stability of shear flows is not an issue there. The shear in the tachocline, however, is latitudinal and radial and may be subject to shear-flow instabilities. If the tachocline is turned into a turbulent layer, a problem arises with the mixing of elements, most notably with lithium, which will be destroyed in nuclear fusion up to 0.68 solar radii $\left(R_{\odot}\right)$, just below the convection zone. A turbulent tachocline mixing the lithium into its fusion zone would contradict the observed relatively high lithium abundance at the surface of the Sun.

The situation in the solar tachocline was described by Spiegel \& Zahn (1992) as exhibiting horizontal turbulence, excited by hydrodynamic shear-flow instability. It was argued that the two-dimensional turbulence provides angular momentum transport but inhibits too strong a mixing of lithium below $0.68 R_{\odot}$.

The hydrodynamic stability of the latitudinal shear in the tachocline was studied by Watson (1981). The dependence on colatitude $\theta$ was $\Omega=\Omega_{\mathrm{eq}}\left(1-\alpha_{2} \cos ^{2} \theta\right)$, where $\Omega_{\mathrm{eq}}$ is the equatorial angular velocity at the bottom of the convection zone and $\alpha_{2}$ is a parameter for the relative equator-to-pole difference of the angular velocity. Watson found instability for a differential rotation with $\alpha_{2}>0.286$. At that time, this was supposed to be in the vicinity of the value of solar differential rotation, and the result was not definitely decisive between a turbulent and a stable tachocline. The investigation was two-dimensional, arguing that the stable stratification will not allow significant radial flows, but the radial shear is thereby neglected, too.

Charbonneau et al. (1999a) extended the linear stability analysis to various rotation profiles of the form

$\Omega=\Omega_{\mathrm{eq}}\left(1-\alpha_{2} \cos ^{2} \theta-\alpha_{4} \cos ^{4} \theta\right)$.

Different layers of the tachocline were associated with different results from helioseismology in terms of $\alpha_{2}$ and $\alpha_{4}$. The upper layer, which is thought to be penetrated by convective overshooting, was found to be unstable to the shear, whereas the lower layer with smaller latitudinal shear turned out to be stable. In a step towards the three-dimensional stability of the tachocline, Dikpati \& Gilman (2001) studied the two-dimensional system allowing for deformations in the third - the radial - dimension. As a result, the critical differential rotation for instability was reduced to $11 \%$ in the overshoot part of the tachocline.

In order to address the entire tachocline, because the tachocline is a place where latitudinal and radial shear meet, we investigate the stability of the three-dimensional rotation profile. Although it is very reasonable to assume that radial flows will be weak, the variation of the latitudinal differential rotation with radius across weakly coupled spherical layers could provide different results for the stability of the tachocline. We will 
briefly summarize the numerical background in Sect. 2, provide details of the computational results in Section 3 with different rotation profiles, and summarize our findings in Sect. 4. We find that the tachocline is hydrodynamically stable in all the configurations studied. This paper is thus intended to be a preparatory step towards fully three-dimensional studies of the MHD stability of the tachocline.

\section{Computational setup}

The rotational profile depends on both latitude and radius in this study. Between the inner and outer radius of the tachocline, $r_{\mathrm{i}}=0.65$ and $r_{\mathrm{o}}=0.7$, respectively, the angular velocity is defined by

$\Omega(r, \theta)=\Omega_{\mathrm{eq}}\left[1-\alpha_{2} \cos ^{2} \theta-\alpha_{2}\left(\frac{1}{4}-\cos ^{2} \theta\right) \frac{r_{\mathrm{o}}-r}{r_{\mathrm{o}}-r_{\mathrm{i}}}\right]$,

where $\theta$ denotes the colatitude in the spherical shell, $r$ the radial coordinate, and $\Omega_{\mathrm{eq}}$ the equatorial angular velocity. The profile implies that the rotation velocity at the inner boundary of the computational domain, $r=r_{i}$, is the one at $\theta=60^{\circ}$ (or $30^{\circ}$ heliographic latitude). This appears to be a valid assumption in agreement with various helioseismological inversions (recently e.g. Schou et al. 2002), whereas core rotation may be adopted at larger latitudes higher up in the convection zone. The formation of the tachocline rotation profile is supposed to be caused both by rotating convection on top of it and by reduction of differential rotation by magnetic fields in the solar core at the bottom. Such a profile causes a meridional circulation reaching steady-state in competition with the aforementioned (or other tachocline-forming) effects (see e.g. Sule et al. 2005). In our linear analysis, this flow has no effect on the stability of the non-axisymmetric modes investigated in this paper.

We employ the incompressible, viscous Navier-Stokes equation and linearise the problem. We can then separate the axisymmetric background rotation $\boldsymbol{U}$ from the nonaxisymmetric flow $\boldsymbol{u}$. The latter is evolved by numerical computations. The normalised equation of motion reads

$$
\begin{aligned}
\frac{\partial \boldsymbol{u}}{\partial t}= & \boldsymbol{u} \times \nabla \times \boldsymbol{U}+\boldsymbol{U} \times \nabla \times \boldsymbol{u} \\
& -\nabla p-\nabla(\boldsymbol{u} \cdot \boldsymbol{U})+\Delta \boldsymbol{u}
\end{aligned}
$$

and the continuity equation $\nabla \cdot \boldsymbol{u}=0$ holds. The equation is evolved with the spectral spherical code by Hollerbach (2000). We look for exponentially decaying or growing solutions in order to find the critical differential rotation $\alpha_{2}$ of the marginal case. The actual integration employs the radial components of the curl and the curl-curl of the equation, thereby eliminating the gradient terms. The linear (viscous) part of the full equation of motion is evolved implicitly, while the nonlinear parts are integrated explicitly with the advection term and the forces being computed in real space. We kept this splitting even in our linearised problem, since a fully implicit scheme would have required a substantial modification of the code. The $\Delta \boldsymbol{u}$ is thus treated implicitly, the other rhs terms (two remaining after curling) are computed in real space and are used for a second-order Runge-Kutta integration.

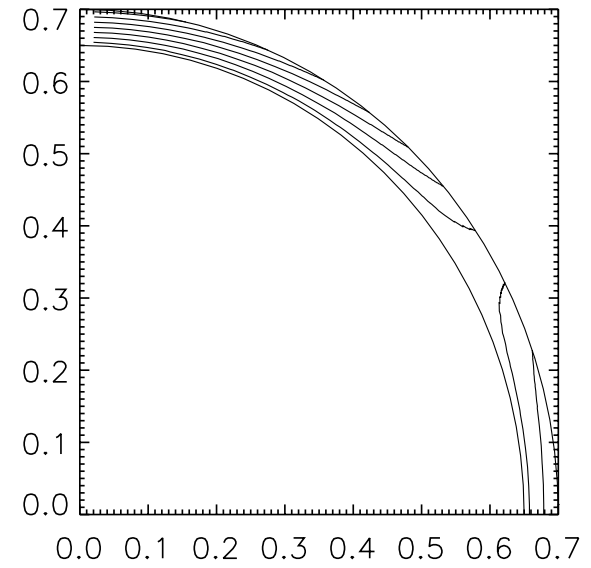

Fig. 1. Vertical cross-section through the solar tachocline with contours of the assumed angular velocity depending on radius and latitude as given by Eq. (2). The highest angular velocity is at the equator (bottom right), and radially constant rotation occurs at a heliographic latitude of $30^{\circ}$.

The normalisation of the equation with the viscous time $\tau_{v}=r_{\mathrm{o}}^{2} / v$ and the length scale $r_{\mathrm{o}}$ leads to the Reynolds number

$\operatorname{Re}=\frac{r_{\mathrm{o}}^{2} \Omega_{\mathrm{eq}}}{v}$

as a free parameter, which is essentially a variation of the viscosity $v$ since radius and $\Omega_{\mathrm{eq}}$ are sufficiently well known. The solar Reynolds number in the tachocline is - in terms of the definition of Eq. (4) - about $10^{14}$. We try to achieve time series for numerically demanding $\operatorname{Re}>10^{4}$. By comparison with known results from inviscid two-dimensional analyses, we find that the critical viscous differential rotation at $\operatorname{Re}>10^{3}$ or $10^{4}$ is already sufficiently close to the inviscid value.

The velocity is decomposed into toroidal and poloidal potentials, $\boldsymbol{u}=\nabla \times(e \hat{\boldsymbol{r}})+\nabla \times \nabla \times(f \hat{\boldsymbol{r}})$, where $\hat{\boldsymbol{r}}$ is the unit vector in radial direction. The potentials are again decomposed into radial Chebyshev polynomials and spherical harmonics. Since it is potentials being evolved, the continuity equation is fulfilled automatically. The density is constant throughout the computational domain. In a thin shell of $5 \%$ of the solar radius, this is a reasonably good approximation of the true situation. We also do not take any deformation of the tachocline into account. Top and bottom radii of the tachocline are constant over latitude. Charbonneau et al. (1999b) found a prolate tachocline with the equatorial part located at slightly smaller radius than the polar end. We assume that the difference of $3.5 \%$ in location of the tachocline has negligible effect on the results, but this may be a question for future investigations.

The radial boundary conditions for the velocity perturbations are stress-free at both $r_{\mathrm{i}}$ and $r_{\mathrm{o}}$. At Reynolds numbers of $\operatorname{Re}>10^{4}$, high spectral resolution was necessary to obtain reliable results. Up to 80 Chebyshev and 80 Legendre polynomials were used to resolve the flow properly.

The azimuthal modes of the problem described by Eq. (3) are decoupled, and we can study the stability of individual $m$-modes separately. Moreover, even and odd latitudinal modes (symmetric and antisymmetric modes with respect to the equator) decouple, and we will have a look into the critical differential rotation for the excitation of instability of the two kinds 


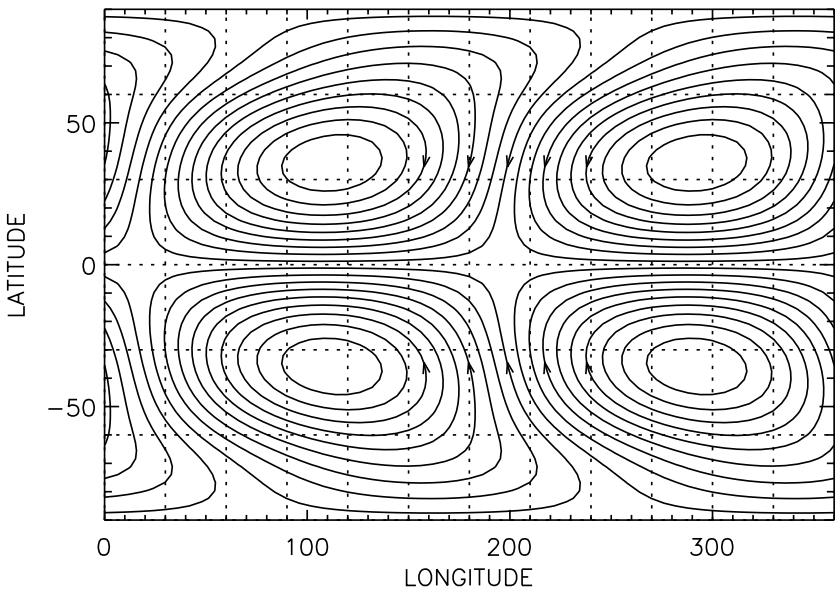

Fig. 2. Streamlines of the symmetric eigen function of the computation with a three-dimensional profile $\Omega(r, \theta)$ on the surface of the tachocline at $r=0.7$. Two circulation cells are found on each hemisphere.

separately. The radial modes all couple and do not provide results on the stability of individual radial wavelengths.

\section{Results}

\subsection{Stability of various solutions}

In a set of fiducial computations, we applied a purely latitudinal profile of the angular velocity. An $m=1$ mode is evolved with the profile of Eq. (1), where $\alpha_{4}=0$ and $\alpha_{2}$ is varied. Since we solve a viscous problem, the critical differential rotation, $\alpha_{2}^{\text {crit }}$, depends on the Reynolds number. The result is already very close to Watson's inviscid result for $\operatorname{Re} \geq 1000$. This is a good reason to assume that the numerical solutions reaching $\operatorname{Re} \sim$ 30000 are suitable approximations for the solar plasma.

Despite allowing for radial motions, the evolution provides solutions which are nearly toroidal and do not show significant radial flows. They are surface flows forming two cells on each hemisphere with stream lines through the poles. Figure 2 shows a representation of the flow in the spherical surface. The graph has to assume that the poloidal component of the velocity is zero, though, which is not entirely true.

The second step involved the rotation profile of Eq. (2), for which the critical steepness of the differential rotation, $\alpha_{2}^{\text {crit }}$, is again sought for various Reynolds numbers. Figure 3 shows the lines of marginal stability, i.e. the critical differential rotation, versus Reynolds number for the symmetric $m=1$ mode. The solid line refers to profile Eq. (2), and the dashed line is the latitudinal profile and converges to the result by Watson (1981) for $\operatorname{Re} \rightarrow \infty$.

The most easily excited patterns of $m=1$ are always symmetric with respect to the equator. We can also look for the stability of antisymmetric patterns and find the results shown in Fig. 4. They are stabler than the symmetric configurations with an $\Omega(r, \theta)$ profile. The antisymmetric solutions from the $\Omega(\theta)$ profile have also higher critical differential rotation values than their symmetric counterparts.

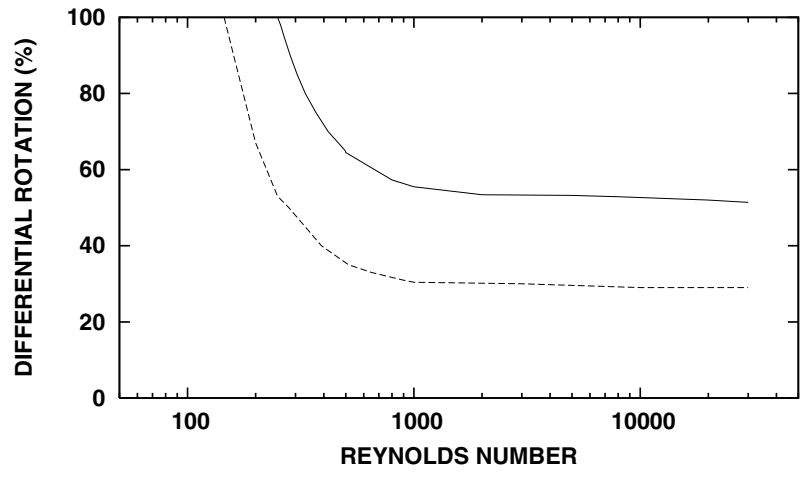

Fig. 3. Lines of marginal stability for the combined latitudinal and radial shear (solid line) and the purely latitudinal shear (dashed line). Differential rotation denotes the percentage by which the pole's angular velocity is slower compared with the equatorial one. It is expressed by $\alpha_{2}$ from Eqs. (1) and (2) here.

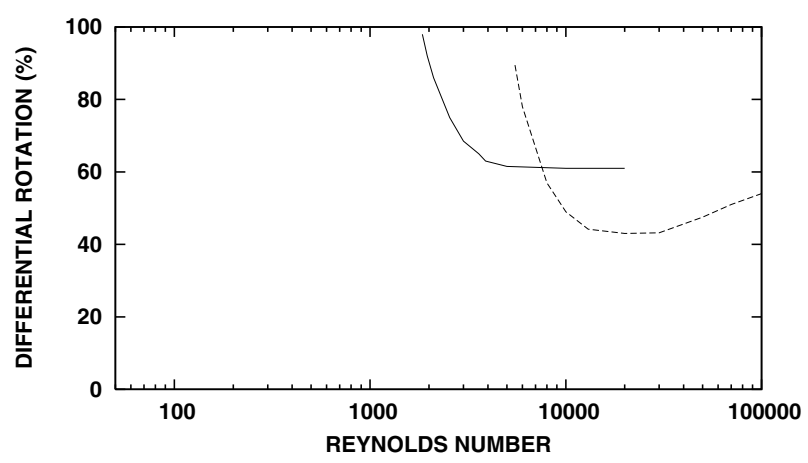

Fig. 4. Lines of marginal stability for the antisymmetric $m=1$ solutions caused by the combined latitudinal and radial shear (solid line) and the purely latitudinal shear (dashed line).

The patterns drift with a certain velocity in azimuthal direction around the solar axis. Since the equations hold for the nonrotating system, we can directly convert the pattern rotation into physical times. The pattern rotation periods for the $2 \mathrm{D}$ and the 3D profiles of $\Omega$ are shown in Fig. 5 by a dashed and a solid line, respectively. The actual solar rotation periods are also plotted. The reference equatorial period of $25.44 \mathrm{~d}$ $\left(\Omega_{\mathrm{eq}}=455 \mathrm{nHz}\right)$ is given as dash-dot line. The pattern rotation periods are determined at marginal stability. Since the marginal case gives us a value for the differential rotation, we also plot the polar rotation period $P_{\text {pole }}$ versus Re. The short-dash line is for the two-dimensional $\Omega(\theta)$ profile, and the dotted line for the three-dimensional case described by Eq. (2).

The pattern rotation periods are always between the equatorial and polar rotation periods, in agreement with the 2D results by Charbonneau et al. (1999a). While the patterns from the $2 \mathrm{D}-\Omega$ profile are close to the polar rotation period, the patterns for the $3 \mathrm{D}$ profile rotate with nearly the average rotation period between the polar and equatorial ones.

We can compute the time after which the pattern is passed by a given point on the equator. This time is often called lap time. Assuming an equatorial rotation period of $25.44 \mathrm{~d}\left(\Omega_{\mathrm{eq}}=\right.$ $455 \mathrm{nHz}$ ), we find a lap time of $91 \mathrm{~d}$ for the $2 \mathrm{D}$ case and a lap time of $78 \mathrm{~d}$ for the $3 \mathrm{D}$ case. 


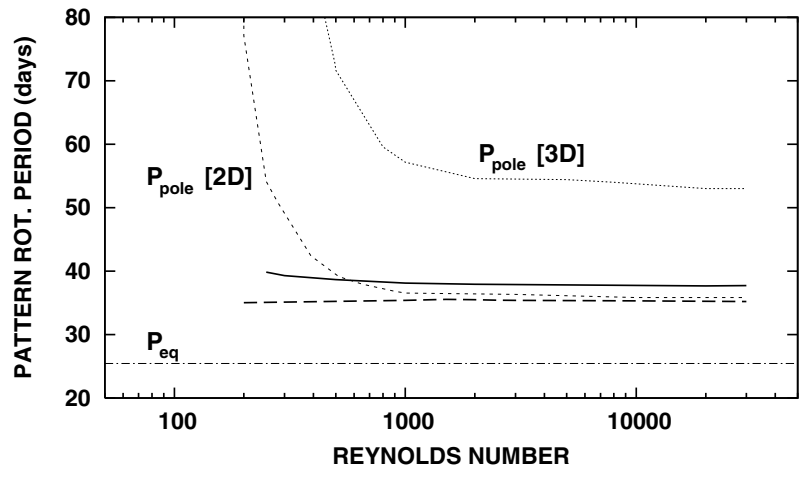

Fig. 5. Rotation period of the flow pattern for the two-dimensional (long-dashed) and the three-dimensional (solid) rotation profile. The numbers are computed assuming an equatorial rotation period of $25.44 \mathrm{~d}\left(\Omega_{\mathrm{eq}}=455 \mathrm{nHz}\right)$ plotted with a dash-dot line. Periods are computed at marginal stability; the polar rotation period for this critical differential rotation is plotted as short-dashed and dotted lines for the $2 \mathrm{D}$ and $3 \mathrm{D}$ cases, respectively.

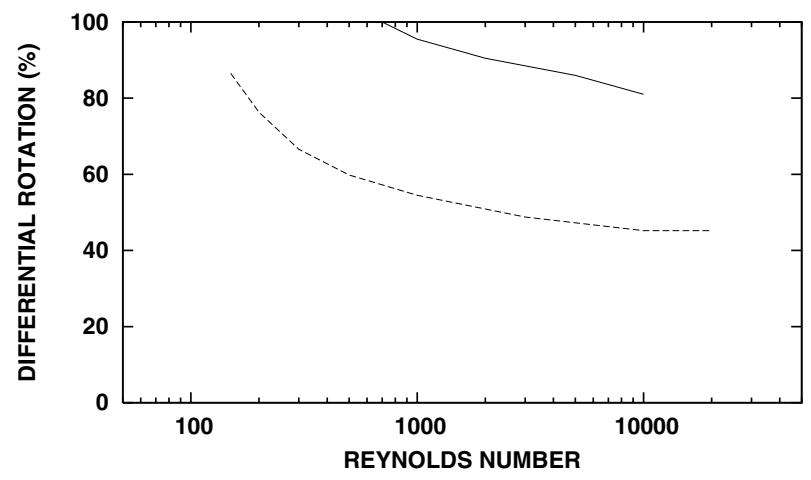

Fig. 6. Lines of marginal stability for the antisymmetric $m=2$ mode resulting from the combined latitudinal and radial shear (solid line) and the purely latitudinal shear (dashed line).

Modes with higher azimuthal mode numbers require significantly higher differential rotation for instability. The antisymmetric $m=2$ mode, which is symmetric with respect to the equator, was found to be stable even in the entire parameter range covered by Fig. 3. This is in agreement with the inviscid, two-dimensional stability analysis by Charbonneau et al. (1999a). The stability lines for the antisymmetric $m=2$ mode are shown in Fig. 6. We could not find instability for any $m=3$ mode in the range covered by Fig. 3 .

\subsection{Effects of buoyancy}

Little influence is expected from the stable temperature gradient in the tachocline. Since we do have the chance to prove this in our three-dimensional simulations, we demonstrate the effect of a negative buoyancy force on the stability of the differential rotation. The Navier-Stokes equation is extended by the buoyancy force and reads in the non-dimensional form as

$$
\begin{aligned}
\frac{\partial \boldsymbol{u}}{\partial t}= & \boldsymbol{u} \times \nabla \times \boldsymbol{U}+\boldsymbol{U} \times \nabla \times \boldsymbol{u} \\
& +\operatorname{Ra} \Theta \boldsymbol{r}-\nabla p-\nabla(\boldsymbol{u} \cdot \boldsymbol{U})+\Delta \boldsymbol{u},
\end{aligned}
$$

$\frac{\partial \Theta}{\partial t}=-\boldsymbol{U} \cdot \nabla \Theta-\boldsymbol{u} \cdot \nabla T+\frac{1}{\operatorname{Pr}} \Delta \Theta$,

with a background temperature profile of

$T=\frac{r_{\mathrm{i}}}{r_{\mathrm{o}}-r_{\mathrm{i}}}\left(\frac{r_{\mathrm{o}}}{r}-1\right)$

and the Prandtl number as the ratio between viscosity and thermal diffusivity, $\operatorname{Pr}=v / \chi$. The Rayleigh number in Eq. (6) is

$\mathrm{Ra}=\frac{g \alpha\left(T_{\mathrm{i}}-T_{\mathrm{o}}\right) r_{\mathrm{o}}^{3}}{v^{2}}$,

where $g$ is the gravitational acceleration, $\alpha$ the coefficient of volume expansion, and $T_{\mathrm{i}}$ and $T_{\mathrm{o}}$ are the temperatures at the two boundaries. In the Boussinesq formulation used here, the presence of a sub-adiabatic temperature gradient actually translates into a negative value of Ra. We set our version of the Rayleigh number to a value as small ("as negative") as Ra = $-10^{8}$ in order to see any notable effect on the flow. The Prandtl number is set to unity.

The critical differential rotation for a growing symmetric $m=1$ mode at $\operatorname{Re}=10^{4}$ increases slightly to $53.3 \%$, as compared with the non-buoyant value of $52 \%$. This is in line with the fact that the solutions contain nearly horizontal motions.

\subsection{Effects of higher-degree terms}

The differential rotation has been expressed by a $\cos ^{2} \theta$ dependence in latitude and a linear $r$ dependence over radius. We are studying the stability of the three-dimensional tachocline setup with higher-degree dependences in this section, such as the $\cos ^{4} \theta$ term and a nonlinear radial dependence of $\Omega$.

Including the $\cos ^{4} \theta$ term in Eq. (2) yields an angular velocity profile of the form

$$
\begin{aligned}
\Omega(r, \theta)= & \Omega_{\mathrm{eq}}\left\{1-\alpha_{2} \cos ^{2} \theta-\alpha_{4} \cos ^{4} \theta-\frac{r_{\mathrm{o}}-r}{r_{\mathrm{o}}-r_{\mathrm{i}}}\right. \\
& \left.\times\left[\alpha_{2}\left(\frac{1}{4}-\cos ^{2} \theta\right)+\alpha_{4}\left(\frac{1}{16}-\cos ^{4} \theta\right)\right]\right\} .
\end{aligned}
$$

The computations for the full profile could not be easily extended beyond $\operatorname{Re}=5000$, but the results for the possible $\mathrm{Re}$ and for the easier two-dimensional $\Omega(\theta)$ show no decreased critical differential rotation when the $\cos ^{4} \theta$ term is included. This also holds for the extreme case of $\alpha_{4}$ carrying the shear alone $\left(\alpha_{2}=0\right)$.

Starting from Eq. (2), a modified $\Omega$-profile was constructed in order to find any influence of the particular radial dependence of the latitudinal shear on the results. We used

$$
\begin{aligned}
\Omega(r, \theta)= & \Omega_{\mathrm{eq}}\left[1-\alpha_{2} \cos ^{2} \theta\right. \\
& \left.-\alpha_{2}\left(\frac{1}{4}-\cos ^{2} \theta\right) \frac{1}{2}\left(1-\cos \left(\frac{r_{\mathrm{o}}-r}{r_{\mathrm{o}}-r_{\mathrm{i}}} \pi\right)\right)\right] .
\end{aligned}
$$

The radial profile introduces two inflection points. It does not apply the usual error function for the radial $\Omega$-step in order to obtain an exact $\partial \Omega / \partial r=0$ at both inner and outer boundary. A graphic representation is shown in Fig. 7. 


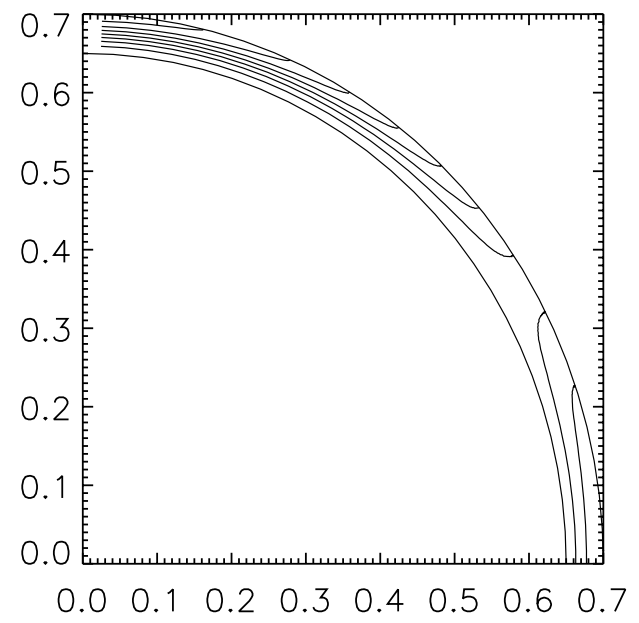

Fig. 7. Contours of the assumed angular velocity with a radial dependence as in Eq. (10). The highest angular velocity is again at the equator (bottom right), and radially constant rotation occurs at a heliographic latitude of $30^{\circ}$.

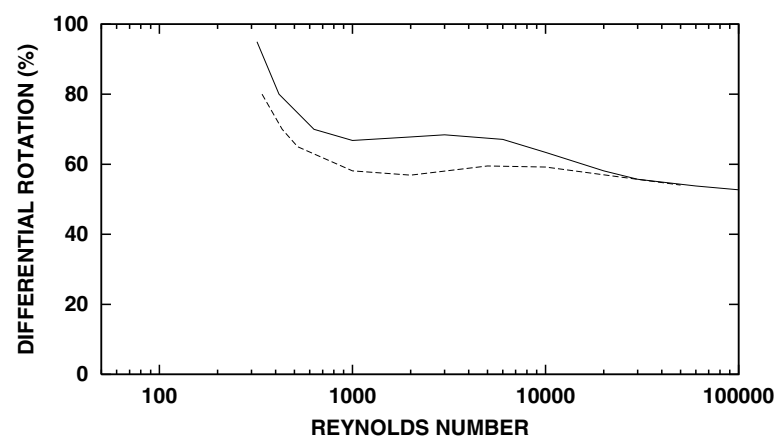

Fig. 8. Lines of marginal stability for an angular velocity profile given by (10) having two inflection points over radius. The solid line refers to the non-buoyant case, $\mathrm{Ra}=0$; the dashed line has $\mathrm{Ra}=-10^{8}$.

The critical differential rotation for instability is shown versus Reynolds number in Fig. 8. We were able to reach extraordinary Reynolds numbers of $10^{5}$, probably because of the vanishing $\partial \Omega / \partial r$ at the boundaries. Instability does not occur at reduced differential rotation, and the line of marginal stability actually converges to the simpler profile (2) for high Re. The additional effect of buoyancy on the stability of the profile (10) with inflection points is also shown as a dashed line.

\section{Summary}

A fully three-dimensional, linear analysis of the stability of the solar tachocline was carried out. If radial variation of the angular velocity is included in the model, the maximum pole-equator difference of the angular velocity can be as large as $52 \%$ for a symmetric $m=1$ mode before instability sets in. Two-dimensional analyses have delivered much lower critical values. The difference between $3 \mathrm{D}$ and $2 \mathrm{D}$ is not radial flows emerging from the extension in the third dimension, but it is the changed stability conditions emerging from the radial shear and radial dependence of the differential rotation.

Other modes, such as higher $m$ or different flow symmetries, do not get unstable at lower critical differential rotation values under the influence of a three-dimensional rotation profile.

The stabilizing effect of the temperature gradient has been added, but since all the unstable modes are very nearly horizontal, the influence is small. The assumption that horizontal motions dominate is valid even without a stabilizing temperature gradient. However the assumption that spherical shells of infinitesimal thickness do not interact with each other is not applicable, according to our results. One may argue that the viscosity in the computer simulations is much too high, but the variation of the results is small at $\operatorname{Re}>1000$. This is an indication that computations with $\mathrm{Re}=10^{4}$ or higher are a good approximation of the near-inviscid solar case. We conclude that all parts of the tachocline not affected by convective overshooting are stable. We believe that the answer to the question of the dynamical state of the tachocline will be given by MHD computations of a three-dimensional domain.

Acknowledgements. We are grateful to Peter Gilman for his valuable comments on the topic. A.S. thanks the Deutsche Forschungsgemeinschaft for the support by grant No. Ru 488/15-1.

\section{References}

Charbonneau, P., Dikpati, M., \& Gilman, P. 1999a, ApJ, 526, 523

Charbonneau, P., Christensen-Dalsgaard, J., Henning, R., et al. 1999b, ApJ, 527, 445

Dikpati, M., \& Gilman, P. A. 2001, ApJ, 551, 536

Hollerbach, R. 2000, Int. J. Numer. Meth. Fluids, 32, 773

Schou, J., Howe, R., Basu, S., et al. 2002, ApJ, 567, 1234

Spiegel, E. A., \& Zahn, J.-P. 1992, A\&A, 265, 106

Sule, A., Rüdiger, G., \& Arlt, R. 2005, A\&A, 437, 1061

Watson, M. 1981, GAFD, 16, 285 